\title{
"SLURRED SPEECH AND TREMORS RESULTING FROM ANTIPSYCHOTIC THERAPY IN A PATIENT WITH BIPOLAR DISORDER: A CASE REPORT"
}

\author{
SOMANABOINA PADMAKAR* \\ Department of Pharmacology, Lovely Professional University, Phagwara, Punjab, India. Email: spadmakar717@gmail.com
}

Received: 08 March 2021, Revised and Accepted: 18 April 2021

\begin{abstract}
Bipolar disorder (BD) is a mood disorder where moods can fluctuate from depression to elevated moods referred to as mania. BD is currently divided into three types. All three types involve clear changes in mood, energy, and activity levels. BD-I is the most severe disorder and symptoms of mania can be so severe that they can require psychiatric hospitalization. BD-II has the same symptoms as BD-I; however, it was described as hypomania because they are less severe than in pure mania. Cyclothymia, also known as a cyclothymic disorder, is a minor mood disorder characterized by fluctuating low-level depressive symptoms and periods of mild mania, similar to BD-II. Mood stabilizers and second-generation antipsychotics are first-line for treating and maintaining a stable mood. This study related to a case report on slurred speech and tremors induced by antipsychotics in a patient suffering from BD.
\end{abstract}

Keywords: Tremors, Bipolar disorder, Cyclothymia, Antipsychotics.

(C) 2021 The Authors. Published by Innovare Academic Sciences Pvt Ltd. This is an open access article under the CC BY license (http://creativecommons.org/ licenses/by/4.0/) DOI: http://dx.doi.org/10.22159/ajpcr.2021v14i6.41341. Journal homepage: https://innovareacademics.in/journals/index.php/ajpcr

\section{INTRODUCTION}

Bipolar disorder (BD) is a mood disorder characterized by recurrent episodes of depression and mania. BD-I and BD-II are the two major subtypes, according to the Diagnostic and Statistical Manual 5. BD-I has a manic episode frequently combined with depression, where BDII has a hypomanic episode often combined with depression [1]. Mood stabilizers, antidepressants, antipsychotic drugs, electroconvulsive therapy, adjunctive medications, and psychosocial therapies are some of the treatment options for BD management. There is evidence that when lithium or valproate is used in addition to antipsychotics to treat acute mania, the effectiveness is higher, and the initiation of action is faster than when used alone. Accordingly, combinations can be used depending on the severity of the mania [2]. Tremor is a trembling sensation caused by an involuntary, rhythmic muscle contraction in one or more areas of the body. Medications that cause tremors include tricyclic antidepressants, monoamine oxidase inhibitors, antipsychotics, lamotrigine, thyroxine, and nicotine. The most common drug-induced tremors are bilateral action tremors [3]. Antipsychotic medications have the ability to disrupt speech processing mechanisms because they affect the neuromuscular system. Speech problems, particularly acquired types, can affect communication and life experiences [4].

\section{CASE REPORT}

A 20-year male patient was admitted to the psychiatry department with chief complaints of abnormal behavior, excessively hungry, paranoid delusion, excessive talking, irrelevant talking, and excessive talking in the past 3 months. He was under medication olanzapine $5 \mathrm{mg}$ and clonazepam $0.25 \mathrm{mg}$. These symptoms were worsened before 1 week as he had stopped taking the medication. Personal history reveals that he had a habit of a mixed diet, sleep disturbances, and decreased appetite, not a known alcoholic/smoker. No relevant family history was found. According to the subjective evaluation and statistical manual of mental disorder, the patient was diagnosed with "BD along with Mania," The patient was treated with the following therapy includes lithium $450 \mathrm{mg} \mathrm{BD}$, sodium valproate $500 \mathrm{mg}$, quetiapine $100 \mathrm{mg}$, haloperidol $5 \mathrm{mg}$, trihexyphenidyl $2 \mathrm{mg}$, diazepam $10 \mathrm{mg}$, olanzapine $10 \mathrm{mg}$, and Inj. haloperidol $2 \mathrm{amp}+$ Inj. Phenergan $1 \mathrm{amp}$. It was continued for 9 days and during the $10^{\text {th }}$ day of the therapy, fresh complaints were noticed they are slurring speech and tremors. During the $13^{\text {th }}$ day of the therapy, other fresh complaints were noticed they are redness of both eyes, pain, and swelling of the left and right gluteal region and the patient was referred to both the ophthalmology and surgery department. The doses for haloperidol were reduced to $5 \mathrm{mg}$. On the $15^{\text {th }}$ day, Inj. fluphenazine $2.5 \mathrm{mg}$ IM stat was added to the prescription continued for 2 days. On day $17^{\text {th }}$ patient was maintaining the same conditions no changes in the complaints. On day 22, haloperidol was discontinued and the patient was discharged with the following medications: Sodium valproate $500 \mathrm{mg}$ twice a day, olanzapine $10 \mathrm{mg}$ twice a day, clonazepam $1 \mathrm{mg}$ twice a day, and advised to attend higher centers for evaluation.

\section{DISCUSSION}

Psychiatric co-morbidity is present in 50-70\% of patients with BDs [5]. Extrapyramidal symptoms and the possibility of tardive dyskinesia are more common with first-generation antipsychotics. Slurred speech, tremors, anxiety, and paranoia are all extrapyramidal symptoms. Second-generation antipsychotics, also known as atypical antipsychotics, have shown direct or indirect benefits in the treatment of anxiety disorders; their additional role as mood stabilizers, which has a generally positive impact on bipolar mood switching, may be advantageous for patients with co-morbidities.

\section{CONCLUSION}

There is a scarcity of evidence for what constitutes best practice in antipsychotic monitoring. The main objectives for the monitoring of antipsychotics are to detect treatable pathology in a high-risk population and to connect and track antipsychotic-induced adverse effects. The caseworker, general practitioner (GP), and psychiatrist are also in charge of physical health monitoring. A monitoring protocol should be implemented into every patient's care plan, according to mental health services.

\section{ACKNOWLEDGMENT}

I would like to express my sincere thanks to Dr. B. Pal, Department of Pharmacology, Lovely Professional University, for inspiring me to write a case study. 


\section{AUTHORS' CONTRIBUTIONS}

Mr. S. Padmakar was involved in data collection, data organization, data interpretation, case study analysis, preparation, reviewing, and editing of the manuscript.

\section{AUTHORS' FUNDING}

There was no specific funding for this case study from any source.

\section{ETHICAL COMMITTEE}

Ethical approval was not applicable to the case report in our institution.

\section{REFERENCES}

1. Maassen EF, Regeer BJ, Regeer EJ, Bunders JF, Kupka RW. The challenges of living with bipolar disorder: A qualitative study of the implications for health care and research. Int J Bipolar Disord 2018;6:1-10.

2. Shah N, Grover S, Rao GP. Clinical practice guidelines for management of bipolar disorder. Indian J Psychiatry 2017;59 Suppl 1:51-66.

3. Kattimani S, Padhy SK, Annamalai I. Unilateral tremor induced by risperidone in a patient with acute mania: Vitamin B12 deficiency as possible mediating factor. Indian J Pharmacol 2012;44:421-22.

4. Sinha P, Vandana VP, Lewis NV, Jayaram M, Enderby P. Predictors of effect of atypical antipsychotics on speech. Indian J Psychol Med 2015;37:429-33.

5. Bobo WV. The diagnosis and management of bipolar I and II disorders: Clinical practice update. Mayo Clin Proc 2017;92:1532-51. 